\title{
Experiment and simulation study on relationship between diameter of high-pressure water abrasive jet nozzle and cutting capacity
}

\author{
Zhang Yu Xian ${ }^{1}$, Li Nan ${ }^{1}$, Wang Hong ${ }^{1}$, Liu Bin Bin ${ }^{1}$ \\ ${ }^{1}$ Guilin University of Electronic Technology, Guilin. Guangxi, China
}

\begin{abstract}
High pressure water abrasive jet nozzle is key part of the particles acceleration. The diameter of nozzle has important effect for jet fluid. Through experiment and simulation explore the relationship between nozzle diameter and jet fluid cutting capacity. The result indicate: (1)the cut depth and broad reduced in linearly relationship with the diameter of the jet nozzle. (2) In same pressure, decrease the nozzle diameter will reduce the cutting ratio energy ;(3)The acceleration and attenuation of the nozzle axial flow in different diameters are basically consistent, The smaller nozzle diameter, The flow acceleration will slower ,the attenuation will faster, the is velocity core segment will shorter and the cutting ability will lower;Consider from energy consumption, cutting efficiency and other factors, for common material cutting the nozzle preferred diameter is: $0.6 \sim 1.0 \mathrm{~mm}$, it's unfavourable to select the $0.2 \mathrm{~mm}$ diameter
\end{abstract}

\section{Introduction}

High pressure water jet technology is a new technique in latest half century, which broad used in cutting, broken and washing. The technique separate with pure water jet and abrasive jet. The nozzle is generation part of AWJ, it's important in abrasive particle acceleration. The nozzle's diameter has deep effect on Jet power and jet machining quality. By experiment and simulation, the article researches the relationship between nozzle diameter and cutting capacity.

\section{The experiment facility and condition}

\section{1 experiment facility}

The experiment use tiny nozzle pro-mix high pressure jet cutting system.(shown in picture 1). The jet float system consist of high pressure pump, abrasive kettle, abrasive jet nozzle ,overflow valve .Throttle and high pressure flexible tube. High pressure water from pump 1separates two aside, one flows into relief valve 7, 8 and another one flows to part 5 . The abrasives through the bottom throttle 9 then flow into mixing cavity 10 and mixture with high pressure water that come from throttle 8. The mixed liquor jet through abrasive nozzle 12.By control the nozzle or work plant can realize cutting workpiece.

When the abrasive jet is required could open the throttle 9, when only use the pure water cut is used to close it. The combined action of 5, 7 and 8 can control the abrasive concentration in the jet stream. When the abradant is needed reduce, close 5,7 part.

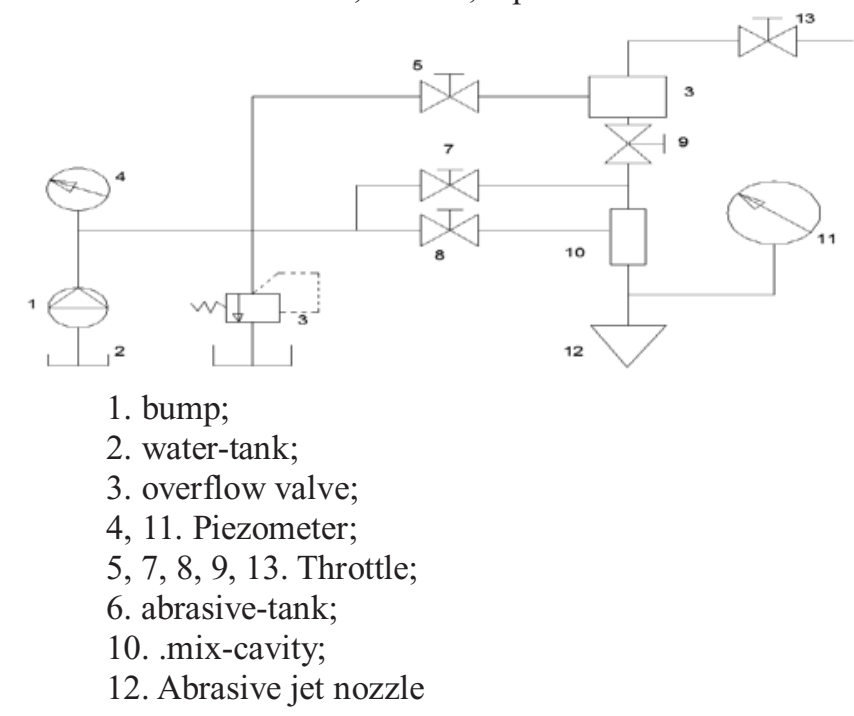

Figure 1. The front mixing abrasive jet system of small nozzles

Under the static water pressure and self-weight effect the abradant arrive mix-cavity 10 .If throttle 7 opening enlarged and the throttle 8 opening reduced, the abrasive concentration will increase accordingly. Throttle 5 is used to adjust the abrasive concentration of the jet flow to meet the cutting requirements. The throttle 13 has exhaust effects. Overflow valve 3 is used to control the working pressure of the system. 


\subsection{The experimental materials}

In this experiment, a variety of brittle and plastic materials were cut, including marble, granite, aluminum and copper plate.
A variety of taper nozzle were used (chart 2), the nozzle entrance is transitioned by arc. Conic section shrinkage angle for $15 \sim 13$, nozzle material is hard alloy, the inner hole surface has the coating, Internal surface quality of nozzle $\mathrm{Ra}$ is $0.8 \mu \mathrm{m}$. The nozzle diameter has $0.2,0.6,1.0$, $1.4,2.0 \mathrm{~mm}$, etc.

\subsection{The experimental nozzle}

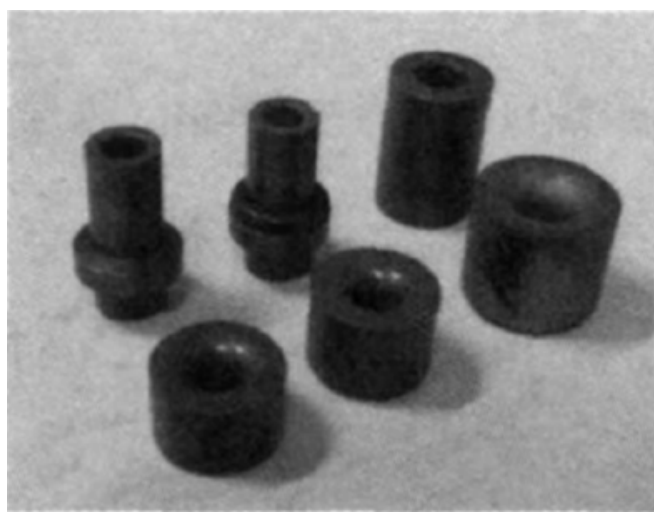

Figure 2. Various experimental nozzles

Figure 3 (a) is the relationship about five different diameter nozzles and correspond cutting depth. The experiment cutting pressure is $15 \mathrm{MPa}$.

\subsection{The influence of nozzle diameter on cutting depth}

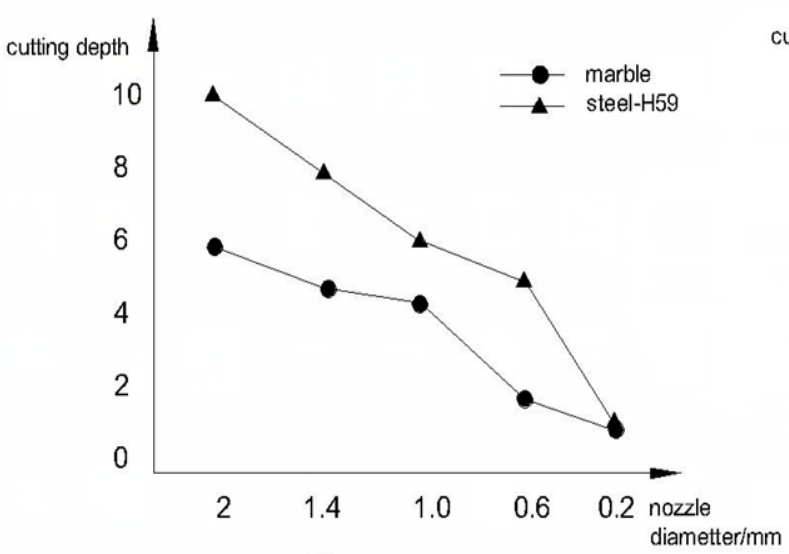

(a)

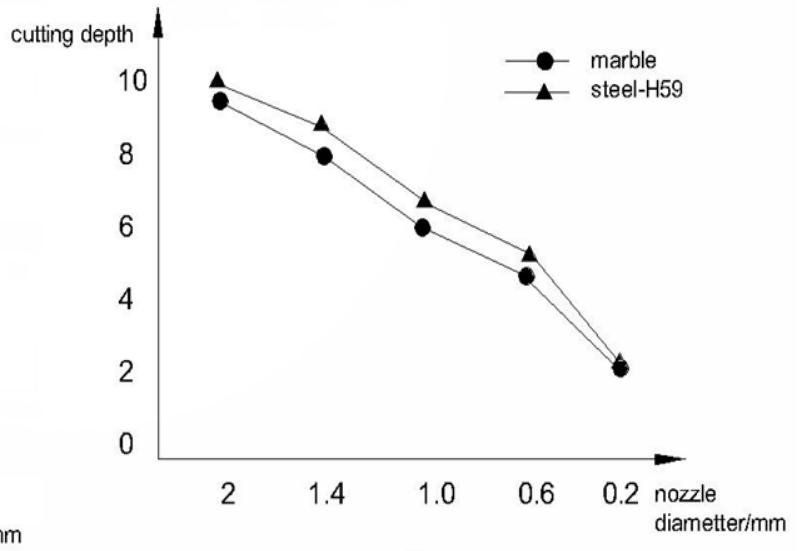

(b)

Figure 3. The incision depth and width of the specimen changed by the nozzle diameter variation

It can be seen that the diameter of nozzle has a significant influence on cutting ability. The cutting depth of marble and brass follow the nozzle diameter changed present similar linearly decreases.

The nozzle $\varnothing 0.6 \mathrm{~mm}$ compared with $\varnothing 2 \mathrm{~mm}$, its cutting ability has decreased observably. When cutting marble, the cutting depth of the former is about $1 / 3$ of the latter, when cutting the copper plate, it is only $1 / 6$. When the nozzle diameter is less than $0.2 \mathrm{~mm}$, the cutting ability of the jet is very low. Therefore, to achieve effective cutting of the $\varnothing 2 \mathrm{~mm}$ nozzle, the driving pressure of the system must be further improved.

\subsection{Influence of nozzle diameter on cutting width}

A single factor correlation between nozzle diameter and incision width of specimen is shown in FIG.3b, the width of specimen incision decreases linearly with the decrease of nozzle diameter basically. Through test, The incision width of specimen is larger than jet diameter. When nozzle diameter $d \geq 0.6 \mathrm{~mm}$. The incision width of the specimen is 1.08 1.27 multiple the jet nozzle diameter; When nozzle diameter $\mathrm{d} \leq 0.6 \mathrm{~mm}$, the incision 
width of the specimen is $1.33 \sim 2.5$ multiple the jet nozzle. When $\mathrm{d}=0.2 \mathrm{~mm}$ the multiple get maximum.

The abrasive water jet cutting material mainly depends on the grinding effect of abrasive particles on the material. On the one hand, the abrasive particle is discontinuous in the water jet. High speed particles composed of abrasive particles also have high frequency impact on materials. The workpiece material is gradually destroyed by the high frequency shock and grinding of high energy abrasive particles. When the nozzle diameter is reduced to $0.2 \mathrm{~mm}$, the boundary layer of jet flow increases the influence of particles. As the complexity of the boundary flow, the increase of the width of the specimen incision is made, another hand, due to the continuity of the specimen material, the particles needed to produce enough energy can tear apart the connections between the specimens. Therefore, when $\mathrm{d} \leq 0.2 \mathrm{~mm}$, through decrease the nozzle diameter to reduce the incision width and improve the stock utilization is meaningless.
Cutting ratio energy consumption is the energy consumed by cutting or crushing unit volume workpiece material, It is the comprehensive quality index of water jet technology, and reflects the rationality and technical level of the system. Its expression is:

$$
E=\frac{\pi \sqrt{\frac{2}{\rho}} p^{\frac{3}{2}} d^{2}}{4 b H v}
$$
value.

$\mathrm{b}$ is the cutting depth, $\mathrm{H}$ is the incision depth, $\mathrm{v}$ is the

The cutting ratio energy consumption of different diameter in simple pressure:

$$
E \frac{E_{2}}{E_{1}}=\frac{H_{1}}{H_{2}} \frac{b_{1}}{b_{2}}\left[\frac{d_{2}}{d_{1}}\right]^{2}
$$

The table 1 shows two different diameter nozzle's cutting ratio energy consumption (cutting material: marble)

\subsection{Cutting ratio energy consumption}

Table 1. The cutting ratio energy about $\varnothing 0.6 \mathrm{~mm}$ and $\varnothing 1 \mathrm{~mm}$ diameter nozzle E2/E1

\begin{tabular}{|c|c|c|c|c|c|}
\hline Pressure(MPa) & 5 & 10 & 15 & 20 & 25 \\
\hline E2/E1 & 0.668 & 0.611 & 0.827 & 0.887 & 0.666 \\
\hline
\end{tabular}

As the pressure increases, the jet cutting ration energy consumption shows decrease tendency. From table 1, in the same pressure, as this nozzle diameter decrease, the ratio of cutting energy consumption is also reducing. In the experiment, the work stress is $5 \sim 20 \mathrm{MPa}$. The cutting ratio energy consumption of $\varnothing 0.6 \mathrm{~mm}$ jet nozzle compared with $\varnothing 0.1 \mathrm{~mm}$ is lower than $11.3 \% \sim 38.9 \%$.

\section{Simulation and analysis of jet nozzle}

\subsection{The simulation conditions}

\subsubsection{The geometric model}

The most critical parts of the efflux are the pipes, nozzles, and the cut workpiece, and the key is nozzle. The Internal surface shape and roughness for the pipe and nozzle has great effect on jet-flow. The geometry parameter in the simulated of this paper have: pipe diameter $8 \mathrm{~mm}$,nozzle diameter $2 \mathrm{~mm}, 1 \mathrm{~mm}, 0.6 \mathrm{~mm}$, $0.2 \mathrm{~mm}$ etc., the target distance $5 \mathrm{~mm}$.

\subsubsection{Fluid condition and numerical simulation environment}

$$
\frac{\partial}{\partial_{t}}\left(\vec{\rho}_{v}\right)+\nabla \llbracket\left(\vec{\rho}_{v}+\vec{v}\right)=-\nabla p+\nabla[\mu(\nabla \vec{v}+\nabla \vec{v} T)]+\vec{\rho} g+\vec{F}
$$

Water: incompressible flow, except boundary layer, flow state is turbulent. Grinding material: Non-crushing solid, density $2600 \mathrm{~kg} / \mathrm{m}^{3}$. Air: room temperature gas. The CFD package is fluent.

\subsubsection{Jet physics model}

The abrasive jet is actually a three-phase jet couple constitutes with water, air and abrasive. Due to the complexity of the problem, this paper only considers the flow of water and air. The abrasive particles are added to the flow field in discrete phase.

Continuity equation:

$$
\frac{\partial a_{q}}{\partial_{t}}+\vec{v} \bullet \nabla a_{a}=0
$$

$\mathrm{V}$ is speed, $a_{q}$ is the volume ratio of q phase fluid. The equation satisfies the constraint $\sum_{q=1}^{2} a_{q}=1$

The momentum equation of the two phases of water and air is:
$\mathrm{P}, \mu, \mathrm{F}$ respectively are average density, viscosity and unit mass of the two-phase fluid.
The turbulent kinetic energy equation (5) of RNG $k-\varepsilon$ model and the diffusion equation (6): 


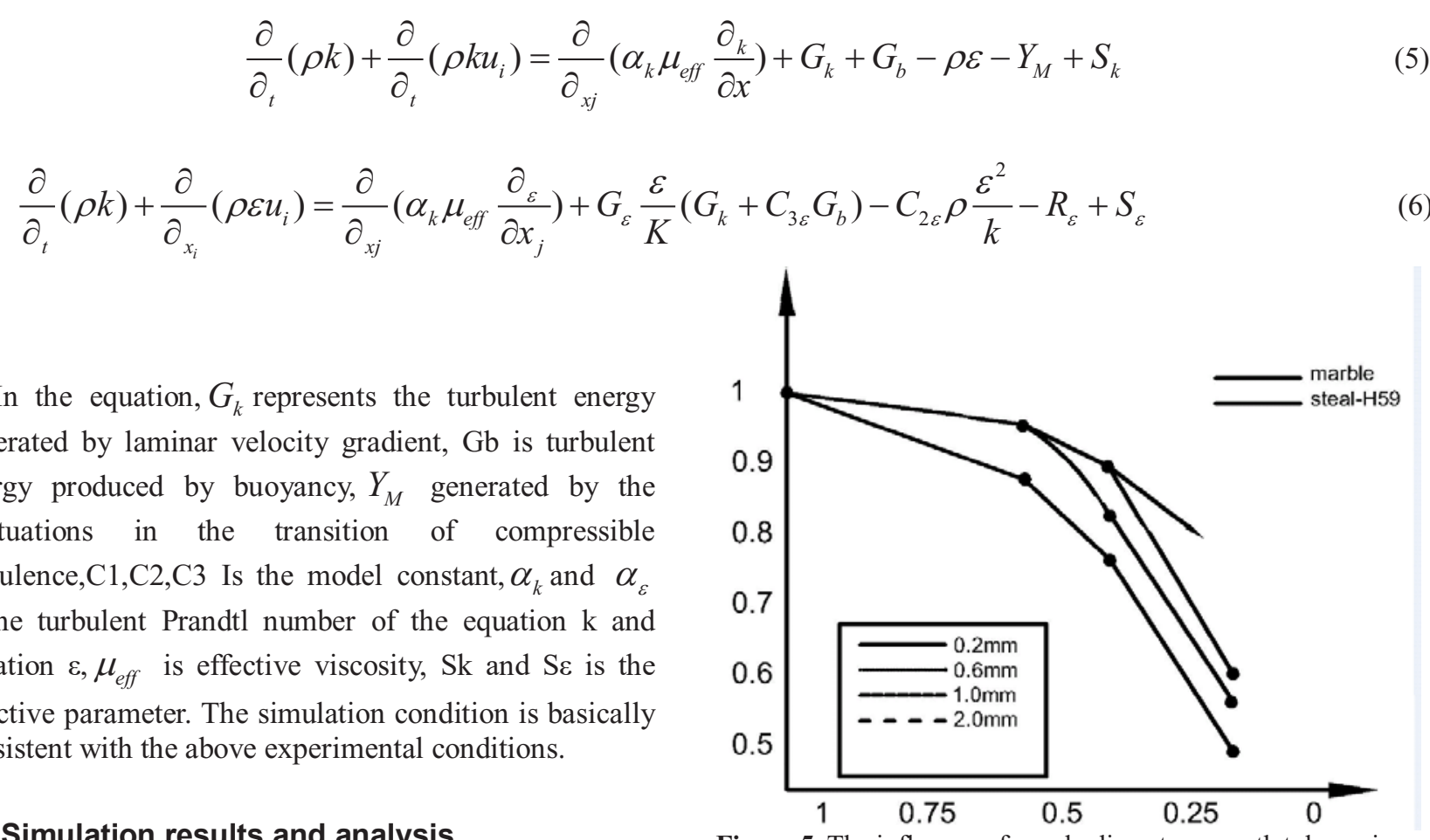

Figure 5. The influence of nozzle diameter on outlet dynamic pressure

If the target distance not changed, the steeper of the curve shows the greater influence of the dynamic pressure on the nozzle diameter. When the nozzle scale among1 0. 5mm (the nozzle diameter is $2 \sim 1 \mathrm{~mm}$ ), the curve flattens out, when the target is increased to $10 \mathrm{~mm}$, The dynamic pressure ratio of efflux still maintains 0.98 . When the nozzle diameter among $0.6 \sim 0.2 \mathrm{~mm}$, the curve section more steeper, the dynamic pressure drops quickly more. At $5 \mathrm{~mm}$ target distance, $0.2 \mathrm{~mm}$ nozzle dynamic pressure ratio is just 0.65.Cutting ability significantly decreased. At the nozzle exit, the dynamic pressure ratio of the $2 \mathrm{~mm}$ nozzle is 0.88 ; therefore, the best target distance is within $5 \mathrm{~mm}$. When the target distance is more than $10 \mathrm{~mm}$, the dynamic pressure ratio of various nozzles is obviously reduced.

When we want the dynamic pressure ratio reaches 0.90 in $5 \mathrm{~mm}$ distance, the nozzle diameter should above $0.28 * 0.2 \mathrm{~mm}=0.56 \mathrm{~mm}$ (using linear interpolation). If the ratio of dynamic pressure reached 0.80 , the nozzle diameter is above $0.18 * 0.2 \mathrm{~mm}=36 \mathrm{~mm}$ approximately.Experiments indicate, using $0.6 \mathrm{~mm}$ nozzle. In the condition of $25 \mathrm{M} \mathrm{Pa}$, the plastic and brittle materials such as copper, aluminum and marble can be effectively cut. Therefore, to achieve effective cutting of above material by $0.5 \mathrm{~mm}$ nozzle, the driving pressure of the system must be increased to more than $25 \times 1 / 0.86 \approx 30 \mathrm{MPa}$.

\section{Conclusions}

The following conclusions can be drawn from the study of cutting experiment and simulation in different nozzle diameters:

(1) The influence of nozzle diameter on cutting ability: The depth and width of the incision are reduced linearly 
by the nozzle diameter .The incision wide of the specimen is larger than the diameter of the efflux, and smaller the diameter is, the more obvious the trend is. When the nozzle diameter is less than $0.2 \mathrm{~mm}$, the jet cutting ability is very low.

(2) The acceleration and attenuation of the different nozzle diameters axial flow are basically consistent. The smaller the nozzle diameter, the jet acceleration will slower, the highest speed is also smaller, but the faster the attenuation, the isovelocity core section get shorter, and the cutting ability get reduced. The best target distance is within $5 \mathrm{~mm}$ ranges. To achieve the effective cutting of the small nozzle $(0.2 \sim 0.5 \mathrm{~mm})$, the driving pressure of the system must be above $30 \sim 38 \mathrm{MPa}$.

(3) Consider the energy consumption, the processed materials saved, cutting efficiency and equipment investment and other factors. The better choice of cutting ordinary material is $0.6 \sim 1.0 \mathrm{~mm}$ nozzle diameter. The $0.2 \mathrm{~mm}$ nozzle is not suitable; under the same pressure, reducing nozzle diameter will decrease the cutting ratio of the jet-flow.

\section{Reference}

1. WANG J X, ZHANG S X. Experimental study on abrasive jet cutting of small nozzles [J]. Fluid machinery St. 2007, 35 (9) : 9- 11.

2. ZHANG B Z, YIN J A, ZHAGN H J. Numerical method of fluid mechanics[M].BEI JING: Mechanical industry press, 2003

3. Li Yang. Numerical simulation of fluid jet in pingement on a wall [A]. Pro jet Report for M E608 Mechanical Engineering [C]. Indiana USA: Purdue University, 2004.

4. LIU S D, HU D, TANG C L, etc. The numerical analysis of the pre-submerged abrasive jet [J].Journal of Hunan university of technology, 2008, 22 (4): 102- 104, 108.

5. ZHANG $\mathrm{S} \quad \mathrm{X}$, ZHANG $\mathrm{Y} \quad \mathrm{X}$, WANG $\mathrm{J} \quad \mathrm{X}$ Experimental study of abrasive water jet particle motion simulation and cutting experiment[J] Mining machinery, 2008, 36( 484): 32-

6. Wang J S, ZHANG S X. Experimental studies on cutting performance of a fine abrasive water jet [A]. Advanced Design and Manufacturing for Sustainable Development [C]. Sydney Australia: ICFDM, 2006. 